\title{
Steering Characteristics of an Autonomous Tractor with Variable Distances to the Waypoint
}

\author{
Sang Cheol Kim ${ }^{\dagger}$, Yeong Gi Hong, Kook Hwan Kim \\ Department of Agricultural Engineering, National Academy of Agricultural Science, Suwon 441-707, Korea
}

\begin{abstract}
Autonomous agricultural machines that are operated in small-scale farmland frequently experience turning and changes in direction. Thus, unlike when they are operated in large-scale farmland, the steering control systems need to be controlled precisely so that travel errors can be minimized. This study aims to develop a control algorithm for improving the path tracking performance of a steering system by analyzing the effect of the setting of the waypoint, which serves as the reference point for steering when an autonomous agricultural machine moves along a path or a coordinate, on control errors. A simulation was performed by modeling a 26-hp tractor steering system and by applying the equations of motion of a tractor, with the use of a computer. Path tracking errors could be reduced using an algorithm which sets the waypoint for steering on a travel path depending on the radius of curvature of the path and which then controls the speed and steering angle of the vehicle, rather than by changing the steering speed or steering ratio which are dependent on mechanical performance.
\end{abstract}

Keywords: autonomous tractor, steering system, variable waypoint

\section{INTRODUCTION}

Agriculture is characterized by diverse and unstructured dynamic environment, and thus the possibility of the introduction of unmanned or automated systems was considered skeptical. However, with the development of machinery, electronics, and software technologies, the scope of unmanned and automated systems has expanded ranging from the stationary automated equipment for controlled agriculture to the autonomous robots for open field cultivation, and the use of state-of-the-art navigation systems (e.g., image processing and GPS) has been generalized.

In fact, the unmanned operation of agricultural machines has been attempted since the 1920s, as shown by the patent application of the steering control device for automated operation by Willrodt (1924) and by circle plowing of Sissons (1939). However, it was not until the early 1960s that guidance cable type unmanned agricultural machines were introduced. In the 1980s, Turk et al. (1988) successfully performed the unmanned operation test of a vision guid-

Received Oct 07, 2013 Revised Oct 25, 2013 Accepted Oct 30, 2013

†Corresponding Author

E-mail: sckim7777@korea.kr

Tel: +82-31-290-1851 Fax: +82-31-290-1860 ance based tractor, and Zhang et al. (1988) proposed an electronic control steering system for automated operation. Thus, many researchers became interested in unmanned agricultural machines. In the late 1990, with the advent of precise agricultural technology based on GPS position information, the autonomous navigation of agricultural machines and the unmanned farm work technology have rapidly developed (Reid et al. 2000). Benson et al. (2003) and Murakami et al. (2006) had made advance in applicability of autonomous travelling device based on vision and image processing as navigation system for farm machinery. In 2004, John Deere commercialized a tractor that is equipped with high-precision Starfire GPS and an auto steering system, which increased the convenience of the operation of manned agricultural machines. Since then, many agricultural machinery manufacturers in the United States and Europe have commercialized agricultural machines equipped with autonomous navigation systems. However, they are mostly targeted at large-scale farms (e.g., more than several hectares), and are not appropriate for the farming environment in Korea, which has small farmland. Especially, for the farming environment in Korea, where the sizes of farmland are approximately $100 \mathrm{~m} \times(30 \sim 100 \mathrm{~m})$ and the planting intervals of crops are generally less than $30 \mathrm{~cm}$ though it varies depending on the type of crops, agricultural machines with autonomous navigation should have a small turning radius, 
and should be able to track planned work paths precisely. Therefore, for the autonomous agricultural machines to be used for farming in Korea, the ability to recognize external environment and the performance of a steering algorithm which can precisely track the path that has been planned based on the perceived environment are very important.

A steering system generally consists of electrical and mechanical elements for controlling the direction of a moving object. To make an agricultural machine perform unmanned farm work, a steering system, which has been manually operated, should be controlled by a control algorithm and electrical signals. An autonomous agricultural machine, which is operated in small-scale farmland, frequently experiences turning and changes in direction. Thus, unlike when it is operated in large-scale farmland, the steering control system needs to be controlled precisely so that travel errors can be minimized.

This study aims to develop a control algorithm for improving the path tracking performance of a steering system through the setting of a variable waypoint, considering the mechanical characteristics of the moving parts and the control characteristics of the electronic control steering system, by analyzing the effect of the setting of the waypoint, which serves as the reference point for steering when an autonomous agricultural machine moves along a path or a coordinate, on control errors.

\section{OVERVIEW OF TEST EQUIPMENT AND METHOD}

\subsection{Design of the Tractor Steering Actuator}

Traditionally, wheeled vehicles have been based on the Ackermann steering mechanism (Morgan, 1958). However, with the development of computer-based design technology, Zhang et al. (1988) devised a method for designing a steering system so that it satisfies the Ackermann geometrybased on kingpin angles and caster angles considering the characteristics of a tractor (Sarhan et al. 2009). In the present study, a tractor with an outer steering angle of \pm 30 degs. was considered, and the steering actuator was made to have a steering angle resolution of less than 1 degree so that the errors from steering could be less than $10 \mathrm{~cm}$ even at the maximum speed of the tractor. For the front wheel, the design objective was to make it rotate the entire range from -35 degs. (left) to +35 degs. (right) within 1.8 seconds, in order to simulate the steering pattern of a skilled tractor operator. To achieve this, a motor and a reducer were selected by calculating the maximum steering torque of the $20-\mathrm{kW}$ tractor, as shown in Eq. (1).

$$
\begin{gathered}
T_{a}=\frac{J_{m}+J_{l}}{g} \times \omega=\frac{J_{m}+J_{l}}{g} \times 2 \pi \times \frac{f_{t} \times 1.8}{g} \times \frac{1}{t_{a}} \\
\left(J_{s}+J_{m}+J_{g}\right) \times \frac{\omega}{g}+P_{b}=184.8 \mathrm{~N} \cdot \mathrm{cm}
\end{gathered}
$$

where,

$\boldsymbol{T}_{\boldsymbol{a}}$ : steering torque $(\mathrm{N} \cdot \mathrm{cm}), \boldsymbol{\omega}$ : angular acceleration of the $\operatorname{motor}\left(\mathrm{rad} / \mathrm{s}^{2}\right)$,

$f_{i}$ : pulse frequency of the motor $(\mathrm{Hz}), \boldsymbol{t}_{\boldsymbol{a}}$ : system control period,

$\boldsymbol{J}_{\boldsymbol{m}}$ : moment of inertia of the motor $\left(\mathrm{N} \cdot \mathrm{cm}^{2}\right), \boldsymbol{J}_{\boldsymbol{l}}:$ moment of inertia of the load side $\left(\mathrm{N} \cdot \mathrm{cm}^{2}\right)$

$\boldsymbol{J}_{\mathbf{g}}$ : moment of inertia of the reduction gear $\left(\mathrm{N} \cdot \mathrm{cm}^{2}\right)$,

$\boldsymbol{J}_{\boldsymbol{s}}$ : moment of inertia of the power steering valve $\left(\mathrm{N} \cdot \mathrm{cm}^{2}\right)$,

$\boldsymbol{P}_{b}$ : back pressure constant of the power steering valve

For the driving motor of the actuator, a full-step twophase step motor was used. To satisfy the design objective (a resolution of less than $1^{\circ}$ ), the pulse frequency $\left(\boldsymbol{f}_{t}\right)$ was set to $1 \mathrm{Kpps}$, the step angle $(\theta)$ was set to $1.8^{\circ}$, and the motor rotation angle per 1 pulse was set to $0.18^{\circ}$. As the number of revolutions of the stepping motor was $10 \mathrm{rps}$ and a 3:1 reducer was used, the pulse number of the stepping motor per $1^{\circ}$ steering angle was measured to be about 150 , and the acceleration/deceleration delay time (ta) was measured to be about 0.019 seconds.

\subsection{Manufacture of the Tractor Steering Actuator}

As shown in Fig. 1, the steering actuator was manufactured so that the driving force of the step motor could be transmitted to the steering shaft through the pulley and belt, and so that the manipulated quantity of the steering valve could be detected through the rotary encoder. The driving by the steering wheel or by the motor could be selected by manipulating the belt clutch. For the motor driver, an H-bridge circuit was constructed using four power transistors, and the motor could be driven depending on the

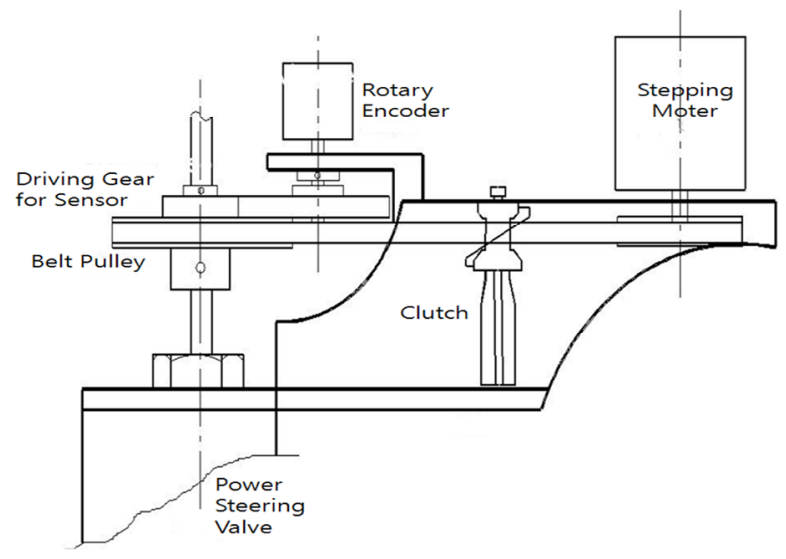

Fig. 1. Schematic diagram of the steering actuator mechanism. 


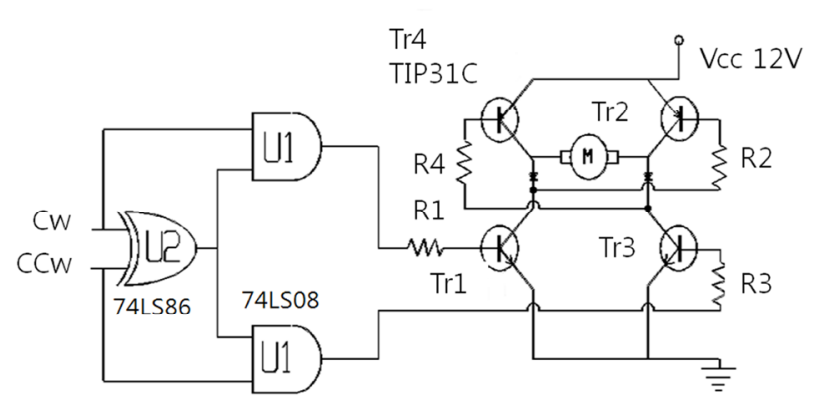

Fig. 2. Circuit diagram of the steering motor driving.

forward/reverse signals using a logic gate. Fig. 2 shows the circuit.

\subsection{Design of the Simulator}

The path tracking accuracy of tractors varies significantly depending on the vehicle speed, steering ratio, and the distance to the steering target point, even though the same steering mechanism is used. A lot of tests have been performed on the effects of vehicle speed and steering ratio on travel errors by various researchers including Xiaopeng et al. (2011). However, the effect of the distance to the steering target point on path tracking errors has not yet been investigated. In this study, a simulation was performed to examine the effect of a variable steering target point depending on the radius of curvature of a path, on the accuracy of path tracking, based on the fact that a tractor operator keeps an eye on a close point when the steering angle is large whereas an operator keeps an eye on a distant point when the steering angle is small, and to make an algorithm for steering control using the obtained results. To investigate the effect of the control speed, optimal steering ratio, and steering target point of a steering system, the mechanical driving parts of a $20-\mathrm{kW}$ tractor were modeled, and the tractor was made to travel the planned paths based on the equations of motion. For the traveling simulation, a two-dimensional plane (Fig.3) was used, and it was assumed as follows.

1) The differential gear system of the tractor operates ideally in relation to the turning radius.

2) Slips in the moving direction and the lateral direction are not considered.

3) The maximum steering angle of the wheel was limited to $35 \mathrm{degs}$ left/right, and the maximum travel speed was limited to $23 \mathrm{~km} / \mathrm{h}$.

For the two-dimensional coordinate transformation of a moving object, the equations of motion for the traveling of

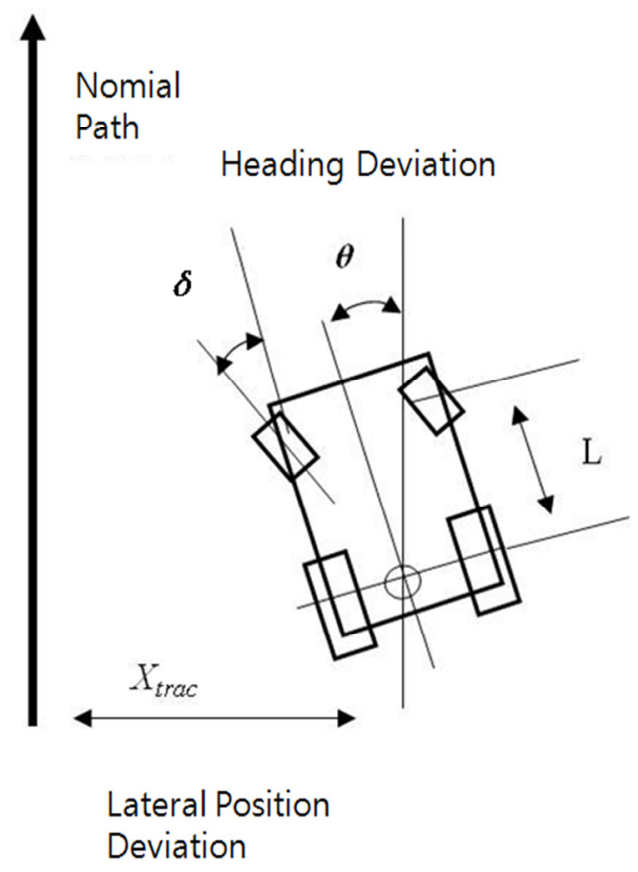

Fig. 3. Lateral position and tractor heading control algorithm.

the tractor were applied to Euler's rotation matrix Gonzalez \& Woods (1993), and the process is as follows. When the tractor travels a distance of $p(\mathrm{~m})$ at a steering ratio of $a$ $(\mathrm{rad} / \mathrm{m})$, the displacements of the center of the tractor front wheel in the $\mathrm{X}$ - and $\mathrm{Y}$-axis directions are

$$
\begin{aligned}
& x=p \cos \left(0.5 \alpha z+\delta_{i}+\theta_{i}\right) \\
& y=p \sin \left(0.5 \alpha z+\delta_{i}+\theta_{i}\right)
\end{aligned}
$$

and the $\mathrm{X}$ and $\mathrm{Y}$ coordinates of the modeled tractor on the two-dimensional plane are

$$
\begin{gathered}
{\left[\begin{array}{l}
X_{i+1} \\
Y_{i+1}
\end{array}\right]=\left[\begin{array}{lll}
1 & 0 & X_{i} \\
0 & 1 & Y_{i}
\end{array}\right]\left[\begin{array}{l}
x \\
y \\
1
\end{array}\right]} \\
\theta_{i+1}=z \frac{\left(0.5 \alpha z+\delta_{i}\right)}{L}+\theta_{i} .
\end{gathered}
$$

Thus, the rotation angle ( $\mathrm{Re})$ of the X-Y plane centered on the Z-axis based on the four-dimensional Euler transformation can be expressed as Eq. (5), and the rows and columns that are not relevant to the X-Y plane of the coordinate system were set to 0 .

$$
R_{e}=\left[\begin{array}{cccc}
\cos \theta & \sin \theta & 0 & 0 \\
-\sin \theta & \cos \theta & 0 & 0 \\
0 & 0 & 1 & 0 \\
0 & 0 & 0 & 1
\end{array}\right]
$$

where,

$$
z \text { : travel distance of the tractor during steering }(\mathrm{m})
$$


$\delta$ : steering angle of the front wheel relative to the center axis of the tractor (rad)

$\boldsymbol{\theta}$ : angle of the center axis of the tractor (rad)

$\alpha$ : steering ratio $(\mathrm{rad} / \mathrm{m})=$ steering angle $(\mathrm{rad} / \mathrm{s}) /$ speed of the tractor $(\mathrm{m} / \mathrm{s})$

$\boldsymbol{L}$ : distance between the centers of the front and rear wheels $(\mathrm{m})$

The maximum steering angle depending on the speed is the maximum steering angle at which the tractor can stably turn at a given travel speed, and it can be obtained from the relation between the overturning moment and the stabilizing moment with the Eq. (6).

$$
\frac{W v^{2} y_{s}}{g} \geq W x, \quad r=L / \sin \theta
$$

where,

$\boldsymbol{W}$ : weight of the tractor $(\mathrm{N})$, g: gravitational acceleration $\left(\mathrm{m} / \mathrm{s}^{2}\right)$

$v$ : speed of the tractor $(\mathrm{m} / \mathrm{s}), \mathrm{r}$ : turning radius $(\mathrm{m})$

$\boldsymbol{y}_{\boldsymbol{s}}$ : vertical distance between the ground and the center of gravity $(\mathrm{m})$

$\boldsymbol{x}$ : horizontal distance between the tread and the center of gravity $(\mathrm{m})$

$\boldsymbol{L}:$ distance between the front and rear axles (m)

During traveling, the steering angle was limited by setting the maximum steering angle considering speed, with the use of Eq. (7).

$$
\theta=\arcsin \left(K \frac{L}{v^{2}}\right)
$$

As for the error range of the path, an error of $5 \mathrm{~cm}$ was allowed including the RMS error of the sensor. For the shortest distance on the path, a certain distance ahead of the current position was designated as the target point on the path. When the allowable error range was exceeded, the tractor was made to move into the path using the maximum steering angle, and the steering angular speed was controlled in proportion to the steering ratio.

\subsection{Construction of the Tester}

To test the performance of the steering control system, a virtual tester was constructed as shown in Fig. 4 . For the tester, the hardware-in-the-loop simulation (HILS) method, which includes electric actuators and sensors, was applied. Software was developed so that it could change the path and speed of the tractor, and then it was interfaced with

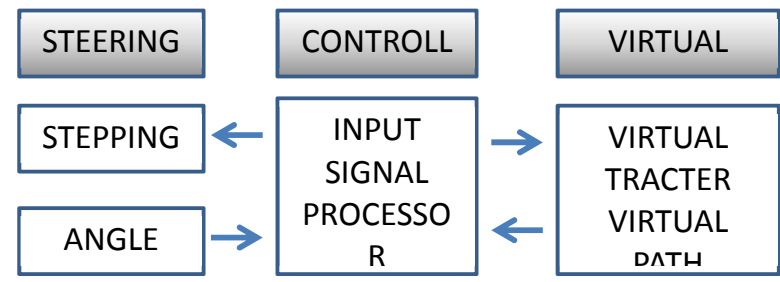

Fig. 4. Block diagram of the virtual tester.

the steering actuator and rotary encoder developed in this study. As for the sensor for detecting the motion of the steering control system while the virtual tractor travels along the sinusoidal and crank-shaped virtual paths (Fig. 5), a rotary encoder (300 pulses/revolution) was used. The motor controller consisted of an Intel 80C51 microprocessor, an input/ output device, and a driver. For the virtual tester, a personal computer (Core2 2.4 GHz) was used.

\subsection{Test Method}

The difference between the route path and the actual trace of the tractor was regarded as the error, while the modeled tractor travels the virtual path as it inputs and outputs signals through the prototype of the steering control system using the tractor's speed, steering ratio and the distance to a target point as the variables. For the distance to a steering target point, a range of $1 \sim 3.5 \mathrm{~m}$ was selected considering the front target point on which a skilled operator keeps an eye in order to travel a given path at a typical working speed of a tractor (mostly $7 \mathrm{~km} / \mathrm{h}$ ). As for the control algorithm, the feed forward control (FFC) for the calculation of target points on a path and the feedback control (FBC) for the correction of errors and the modification of paths using sensor

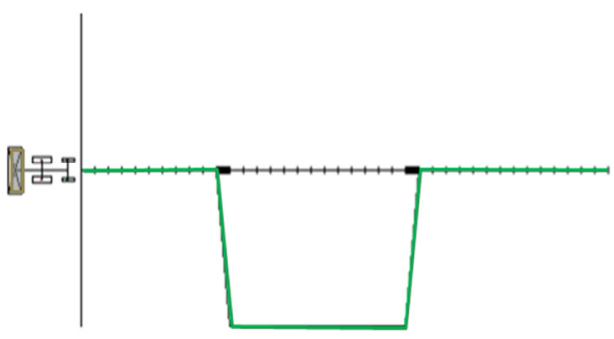

a) Crank-shaped path

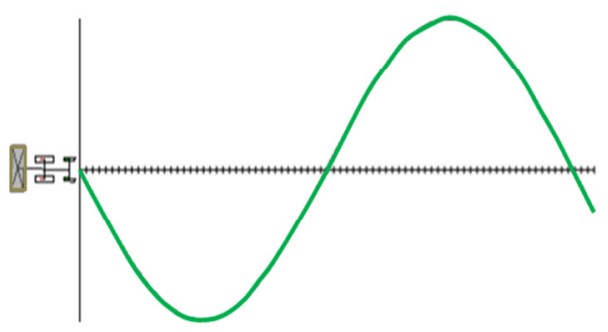

b) Sinusoidal path

Fig. 5. Selected paths for the simulation. 
Table 1. Tested ranges of the tracking control variables.

\begin{tabular}{cc}
\hline Variable & Range \\
\hline Target Point $(\mathrm{m})$ & $0.5 \sim 4.5$ \\
Steering Ratio & $0.08 \sim 0.40$ \\
Speed $(\mathrm{m} / \mathrm{s})$ & $0.2 \sim 6$ \\
\hline
\end{tabular}

signals were utilized at the same time. For the test variables, the ranges of the variables summarized in Table 1 were combined and used.

\section{RESULTS AND DISCUSSION}

\subsection{Performance of the Steering Controller}

In the virtual tester, the path tracking performance of the steering controller was tested using the tractor and the travel path, which had been modeled using software. Thus, there is a limit in investigating the changes in steering characteristics due to the slips in the moving direction or the lateral direction and the sensor errors in the electromagnetic field or vibration environment, which could occur in an actual path. The initialization time of the steering controller was about $3.6 \mathrm{sec}$, and the time for the actuator to reach the time constant depending on the signals was about $23.8 \times 10^{-3} \mathrm{sec}$. The full steering time $(-35 \sim$ +35 degs) could be controlled by changing the input clock of the step motor driver so that it ranged from $1.6 \mathrm{sec}$ to $9.4 \mathrm{sec}$ while maintaining a torque of $180 \mathrm{~N} \cdot \mathrm{m}$.

Fig. 6 shows the traces of the front and rear wheels and the tracking errors, when the tractor travels the sinusoidal path with an amplitude of $6 \mathrm{~m}$, at a speed of $3.6 \mathrm{~km} / \mathrm{hr}$. The path tracking error was obtained using the distance between the center of figure for the projected tractor area on the ground of the two-dimensional model and the predicted passage point on the path. When the radius of curvature of the path changed abruptly, the tractor approached the path with a large error, and the rear wheels showed larger errors than the front wheels. The tracking errors were in the range of $7 \sim 22 \mathrm{~cm}$. Fig. 7 shows the traces of the front and rear wheels and the tracking errors, when the tractor travels the crank-shaped path in the same condition. The errors increased abruptly when the radius of curvature of the path was smaller than the minimum turning radius of the tractor. In the right-angled path, the maximum position error was $128 \mathrm{~cm}$, which was similar to the minimum turning radius.

The test results shown in Figs. 6 and 7 indicated that when the vehicle speed was constant (especially at a low speed) on the same path, the travel error of the same wheel changed slightly depending on the steering speed or steering ratio which are dependent on mechanical performance, but did not show significant difference on the entire path. On the other hand, considering the fact that the rear wheels had larger errors than the front wheels, it will be needed to develop an algorithm which can reduce path tracking errors by controlling the speed and steering angle depending on the radius of curvature of the path.

\subsection{Path Tracking Characteristics by a Variable Waypoint}

Fig. 8 shows the maximum error of the traveling trace at each target point and steering ratio. The horizontal axis represents the distance to the target point, and the vertical axis represents the maximum error between the traveling trace and the planned path. As shown in the figure, the error decreased to a certain degree in the $1.5 \sim 2.0 \mathrm{~m}$ range. However, when the distance to the target point was less than $1.5 \mathrm{~m}$, vibration traveling occurred slightly; and when the distance was more than $2.0 \mathrm{~m}$, it increased abruptly.

As for the proposed steering algorithm, the path tracking error of the tractor varied significantly depending on the distance to the target point on the path, rather than depending on the steering ratio. This is because for the algorithm, which was proposed to improve the test of Rafael et al. (1993) in which the position error varied

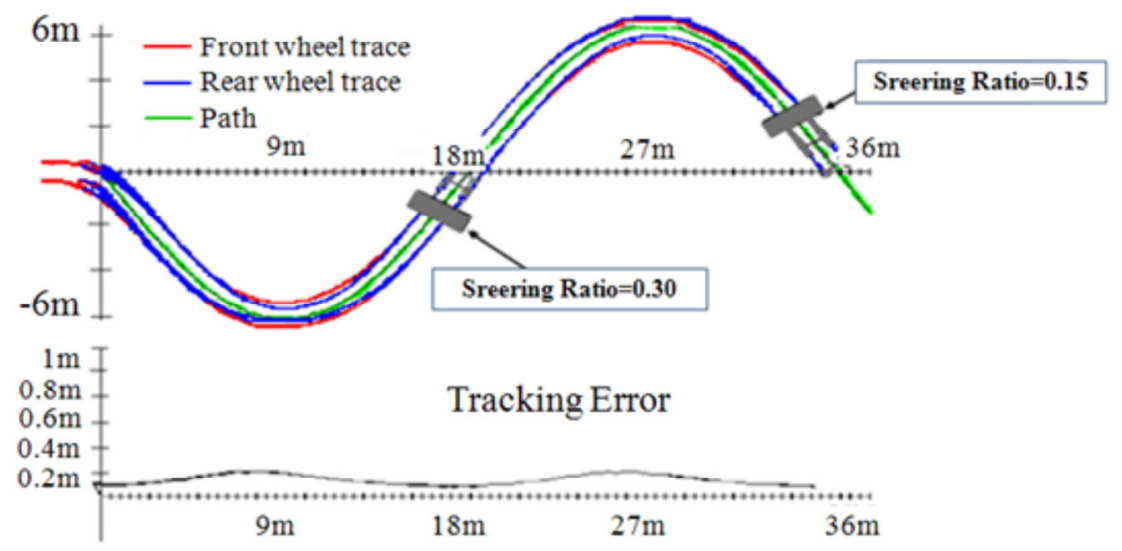

Fig. 6. Tractor trace on the sinusoidal path of the virtual tracing tester. 


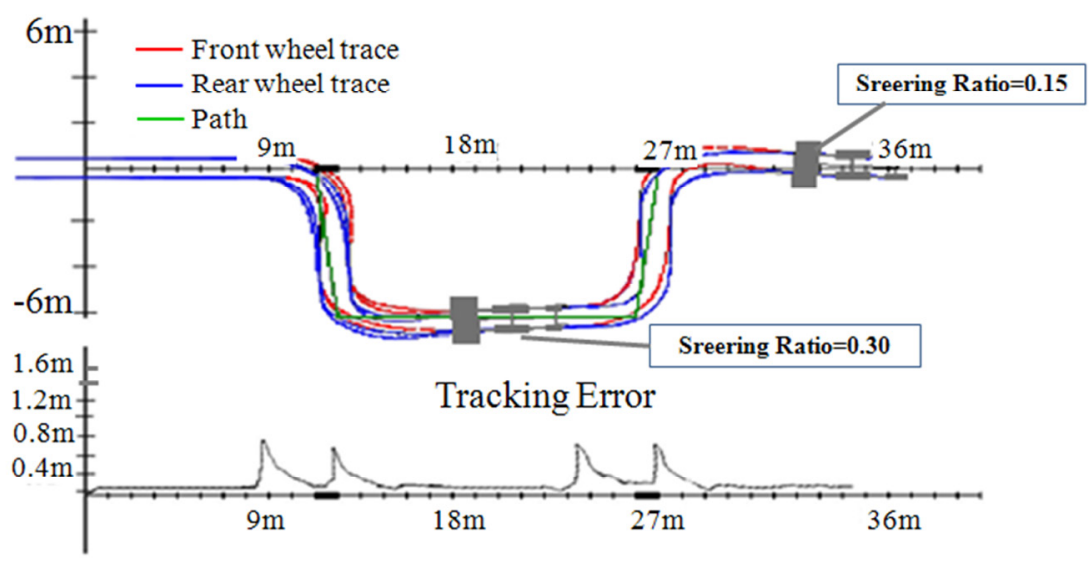

Fig. 7. Tractor trace on the crank-shaped path of the virtual tracing tester.

significantly depending on the steering ratio, the steering algorithm limited the maximum steering angle considering vehicle speed, and the speed was determined by the radius of curvature of the path. The maximum error and the RMS error decreased as the distance to the target point decreased. However, when the target point was too close, vibration traces occurred on the path as the number of operations of the steering actuator increased and the control became unstable.

Fig. 9 shows the RMS error of the traveling trace at each target point and steering ratio. When the distance to the target point was less than $2.0 \mathrm{~m}$, the RMS error was affected by the steering ratio; but when the distance was more than $2.0 \mathrm{~m}$, it was affected only by the distance to the target point. Also, when the distance to the target point was less than 1.5 $\mathrm{m}$, the RMS error increased because of the effect of vibration traveling.

For the steering controller that is operated by electrical signals, path tracking performance as well as the stability of the control system is very important. Based on the results shown in Figs. 8 and 9, the travel error depending on the steering speed or steering ratio which are dependent on mechanical performance did not show significant

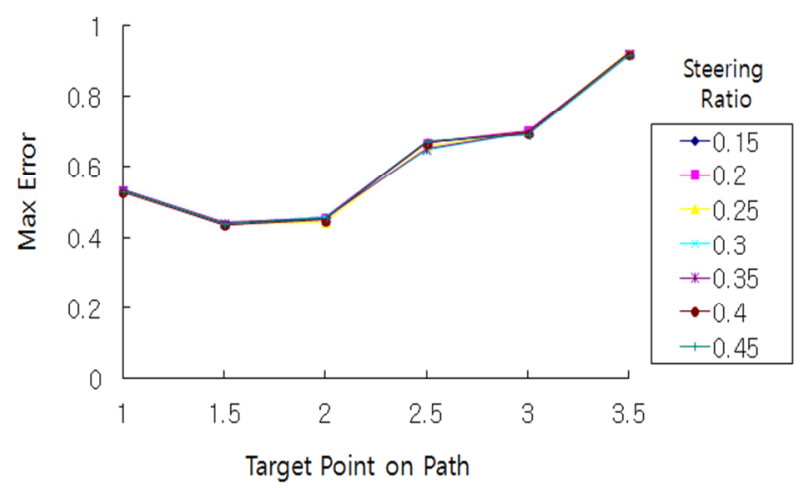

Fig. 8. Maximum error at each target point and steering ratio on the sinusoidal path. difference, whereas the path error showed significant difference when the steering target point changed. Therefore, for changing radius of curvature, the path tracking error of agricultural machines (e.g., tractor) during autonomous traveling could be reduced by developing an algorithm which sets a variable waypoint and controls the speed and steering angle depending on the radius of curvature of the path, which was the objective of this study.

\section{CONCLUSIONS}

For the steering system of an autonomous agricultural machine, to achieve smooth path tracking performance, the steering system needs to be controlled effectively depending on the electrical signals. For the purpose of optimal design, a simulation was performed by modeling a $20-\mathrm{kW}$ tractor steering system and by applying the equations of motion of a tractor, with the use of a computer. In the control algorithm, the speed and steering angle were determined depending on the radius of curvature of the path, and the tractor was made to travel aiming toward the calculated target points on the path. The steering control system was tested

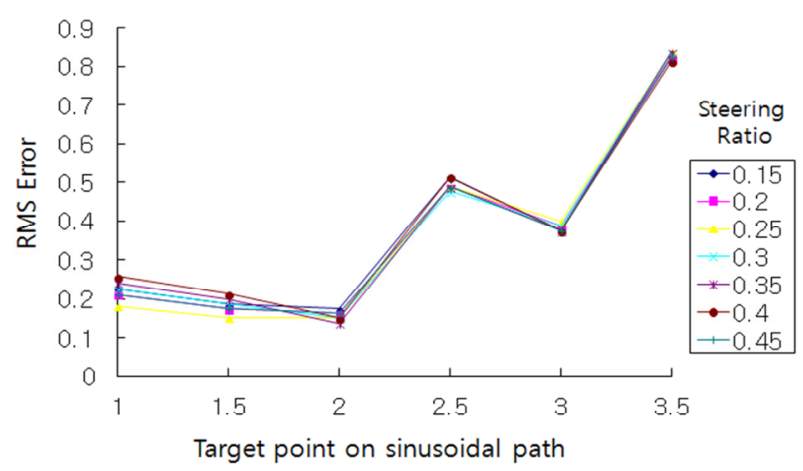

Fig. 9. RMS error at each target point and steering ratio on the sinusoidal path. 
by interfacing it with a computer simulation program which was designed to make the tractor track target points using a tractor steering actuator which was designed so that the steering angle resolution was $0.2^{\circ}$, and so that it could rotate the maximum steering angle $(-35 \sim+35$ degs $)$ within $1.8 \mathrm{sec}$, based on the results of the simulation. The test results can be summarized as follows.

1) The equations of motion of a tractor and the steering mechanism were mathematically modeled, and a simulation was performed using various steering ratios and target points. When the distance to the target point was set to $2 \mathrm{~m}$, the RMS position errors on the sinusoidal path with an amplitude of $6 \mathrm{~m}$ were $11 \sim 14 \mathrm{~cm}$.

2) A virtual tester was constructed using the manufactured steering controller, and it was tested on the sinusoidal and crank-shaped paths. When the tractor travels the sinusoidal path with an amplitude of $6 \mathrm{~m}$, at a speed of $3.6 \mathrm{~km} / \mathrm{hr}$, the tracking errors were in the range of $7 \sim 22 \mathrm{~cm}$. For the traveling of the right-angled path, the maximum position error was $128 \mathrm{~cm}$, which was similar to the minimum turning radius.

3) For low-speed agricultural machines including a tractor, the path tracking errors could be reduced using an algorithm which sets the waypoint for steering on a travel path depending on the radius of curvature of the path and which then controls the speed and steering angle of the tractor, rather than by changing the steering speed or steering ratio which are dependent on mechanical performance.

4) In the future, the steering control system for autonomous traveling will be evaluated and supplemented by constructing an embedded type steering controller based on the results of this study and by performing a test in which a tractor equipped with the constructed steering controller tracks planned work paths depending on the signals of an unmanned navigation system.

\section{ACKNOWLEDGMENTS}

This research was supported by the experimental and research expense of the agricultural basic technology research program funded by National Academy of Agricultural Science (NAAS) of the Rural Development Administration of Korea.

\section{REFERENCES}

Benson, E., Reid, J. F., \& Zhang, Q. 2003, Machine visionbased guidance system for agricultural grain harvesters using cut-edge detection. Biosystems Engineering, 86, 389-398.

Turk, M. A., Morgenthaler, D. G., Gremban, K. D., \& Marra, M. 1988, VITS-A Vision System for Autonomous Vehicle Navigation, IEEE Transaction on Pattern Analysis and Machine Intelligence, 10, 342-361

Morgan, K. E. 1958, A step towards an automatic tractor, Farm Mech, 10, 440-441

Murakami, N., Ito, A., Will, J. D., Steffen, M., Inoue, K., Kita, K., \& Miyarua, S. 2006, Environment identification technique using hyper Omni-vision and image map. In: Proceedings of the 3rd IFAC Intl. Workshop BioRobotics, Sapporo, Japan, pp.317-320

Gonzalez, R. C. \& Woods, R. E. 1993, Digital Image Processing 2nd ed. (New Jersey: Prentice Hall), pp.5161

Reid, J. F, Zhang, Q., Noguchi, N., \& Dickson, M. 2000, Agricultural automatic guidance research in North America, Computers and Electronics in Agriculture, 25, 155-167

Sarhan, A. M. M., Al-Katary, H. S., \& El-Awady, M. N. 2009, Study on agricultural tractors steering mechanism, Misr J. Ag. Eng., 26, 1725-1742

Sissons, R. 1939, Plowing in circles saves time. The Prairie Farmer, 111(20), 7

Willrodt, F. L. 1924, Steering attachment for tractors, U.S. Patent No. 1506706

Xiaopeng, W., Zuoxi, Z., Long, Y., Zhigang, Z., Lian, H., et al., 2011, Tractor Steering Control System Design Basing on Double Closed-loops Control, Intelligent Computation Technology and Automation (ICICTA), 28-29 March 2011 International Conference, pp.294 - 298

Zhang, Y., R. L., Kushwaha, F. W. Bigsby, 1988, Design of common-center-steering systems of tractors. Trans. ASAE, 31, 1015-1019Zhang, Y., Kushwaha, R. L., \& Bigsby, F. W. 1988, Design of common-center-steering systems of tractors, Trans. ASAE, 31, 1015-1019

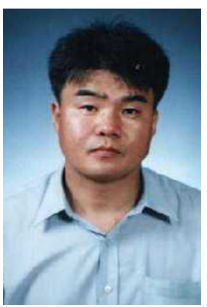

Sang Cheol Kim is a senior researcher of National Academy of Agricultural Science (NAAS) in Korea. He received Ph.D in agricultural machinery at Kongju University. He's been working in the field of agricultural robot development including autonomous travelling technologies of agricultural tractor and transplanter in NAAS since 1997. 


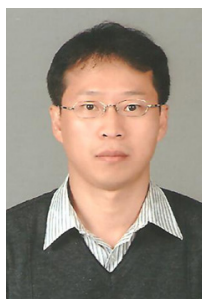

Yeong Gi Hong is a junior researcher of National Academy of Agricultural Science (NAAS) in Korea. He received Ph.D in agricultural engineering at Tokyo Agricultural University in 2004. His research interests include a satellite navigation of agriculture robot, sensor for precision farming and agricultural application of these technologies.

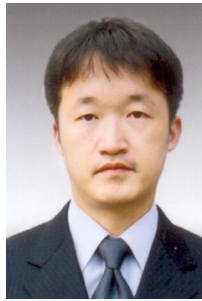

Kook Hwan Kim is a post-doctoral researcher for National Academy of Agricultural Science (NAAS) in Korea. He received Ph.D in mechanical engineering at Kyunghee University in 2010. His research interests include autonomous travelling technologies of field robots 